\title{
Über die Urease der Sojabohne und ihre Verwendung zur quantitativen Harnstoffbestimmung.
}

\author{
Von
}

Fritz Eigenberger, d. Z. im Felde.

(Aus der medizinischen Universitätsklinik von R. v. Jaksch, Prag.)

(Der Redaktion zugegangen am 8. Januar 1915.)

Marschall verwendet in seinem Verfahren zur quantitativen Harnstoffanalyse ein harnstoffspaltendes Ferment der Sojabohne (Glycine hispida), das sich aus dieser durch Wasser ausschütteln läbt.

Auf Veranlassung des Chefs der Klinik, Herrn Hofrat R. v. Jaksch habe ich mich mit dieser Fermentmethode beschäftigt und möchte im Folgenden die Resultate meiner Untersuchungen mitteilen.

Der wässerige Extrakt des Sojabohnenmehls ist milchig trüb, leicht gelblich, opaleszierend, reagiert schwach alkalisch und enthält die Urease. Die chemische Untersuchung der Kolloidstoffe des Extraktes läßt diese als typische Eiweißkörper erkennen, die die wichtigsten für die aliphatischen und aromatischen Eiweißbausteine charakteristischen Gruppenreaktionen geben. Der aromatische Kern läßt sich durch Salpetersäure zu Pikrinsäure nitrieren, wodurch eine leicht zeisiggelbe Färbung auftritt, die auf Kalilaugezusatz in Orange umschlägt. Die Millonsche Tyrosinreaktion ist ziemlich typisch. Nach Pepsinverdauung gibt Bromwasserzusatz eine Rosafärbung, eine Reaktion, die ich bisweilen auch ohne Vorbehandlung mit Pepsin gefunden habe. Nachdem nur freies Tryptophan diese Farbenreaktion bewirkt und ich ferner durch Dialyse stickstoffhaltige Substanzen aus dem Extrakt entfernen und durch das KjeldahlVerfahren nachweisen konnte, so glaube ich, daß im Extrakte eine Reihe freier Aminosäuren enthalten sind. Der Gehalt daran scheint allerdings sehr $\mathrm{zu}$ schwanken und es dürten diese Aminosäuren im keimenden Samen aus den Eiweißmolekülen abgebaut und für die ersten Wachstumsvorgänge zum Transport mobilisiert werden. 
Die Biuretgruppe gibt die charakteristische Farbenreaktion sehr deutlich. Der Cystinkomplex läßt sich durch Ausfall von Schwefelblei nach Zusatz von Kalilauge und Bleiacetat nachweisen. Als Schwefelgehalt fand ich durch Einschmelzen mit Salpeter und Soda und Wägung der durch Baryumchlorid gefällten Sulfate einen Durchschnittswert von $0,8 \%$ für das Trockengewicht der Extrakteiweißstoffe.

Die Kolloide werden aus ihren wässerigen Lösungen durch Siedehitze nicht gefällt. Zusatz geringer Mengen von Lauge bewirkt eine starke Auf hellung unter gleichzeitiger Gelbfärbung der Lösung. Zusatz größerer Mengen wieder Trübung. Genau antogonistisch wirken Säuren: kleine Mengen fällen aus, größere lösen den Niederschlag.

Der durch Kalilauge geklärte Extrakt ist optisch inaktiv oder schwach rechts drehend, nach Ausfällung der Kolloidstoffe dreht er stärker rechts. Diese Rechtsdrehung wird durch hochmolekulare, aber größtenteils dialysierbare Kohlenhydrate bedingt, unter denen mit der Phenylhydrazinprobe Spuren von Traubenzucker nachzuweisen sind. Die übrigen Kohlenhydrate lassen sich weder durch Hydrolyse mit verdünnten Säuren noch durch Ptyalin in einfache Zucker spalten und geben sehr stark die Molisch-Udranskysche Reaktion.

Die Kolloidstoffe des Extraktes lassen sich durch Neutralsalze ausfällen und geben bei Anwendung von Ammonsulfat zwei gut trennbare Fraktionen.

\begin{tabular}{c|c|c}
\hline $\begin{array}{c}\text { Soja- } \\
\text { Extrakt }\end{array}$ & $\begin{array}{c}\text { Kaltgesättigte } \\
\text { Ammonsulfat- } \\
\text { lösung }\end{array}$ & \multicolumn{1}{|c}{} \\
\hline 1 Teil + & $: 1 / 4$ Teil & $\begin{array}{l}\text { gibt trübes, eiweißhaltiges Filtrat. } \\
\text { gibt klares Filträ, das auf Mehrzusatz von } \\
\text { Ammonsulfat oder durch Essigsäure wieder } \\
\text { trüb wird. }\end{array}$ \\
1 Teil + & $1 / 2$ Teil & $\begin{array}{l}\text { gibt das Gleiche. } \\
\text { gibt eiweißfreies Filtrat. }\end{array}$
\end{tabular}

Fällung mit Säure (einigen Tropfen Essigsäure) gibt ein trübes Filtrat, das auf Zusatz von Ammonsulfat weiter getrübt wird und dann klar filtriert.. . 
Man sieht also, daß bereits durch diese rohe Methode zwei Eiweißkörper von etwas verschiedenen Eigenschaften $z u$ trennen sind, von denen der eine, der der wässerigen Lösung die milchig opaleszierende Färbung gibt, durch Ammonsulfat leicht, durch Essigsäure wenig oder gar nicht gefällt wird, während der andere eine klare Lösung bildet, leichter durch Essigsäure und weniger leicht durch Ammonsulfat fällbar ist.

Es erscheint nun sehr fraglich, daß das ganze Eiweißkoagulum, das beispielsweise durch Aceton aus dem wässerigen Sojaextrakt gefällt wird, direkt als harnstoffspaltendes Ferment aufzufassen ist; es handelt sich dabei wohl viel eher um einen ganzen Komplex verwandter Eiweißkörper, die unter entsprechenden Bedingungen gemeinsam ausfallen. Ob das eigentliche Ferment mit einem dieser Eiweißkörper identisch ist, oder ob es unter diesen im chemischen Sinne eine gewisse Sonderstellung einnimmt, konnte ich nicht entscheiden. Es scheint, daß bei Ammonsulfatfällung die erste Fraktion den Harnstoff intensiver angreift als die zweite.

Jedenfalls ist die Urease, wie jedes Ferment ein echtes Kolloid und die strenge Isolierung und chemische Charakterisierung bietet wegen des Mangels an Kriterien und exakten Arbeitsmethoden die gleichen Schwierigkeiten, wie sie sich bei allen Fermentuntersuchungen entgegenstellen.

Für die Technik der Harnstoffbestimmungen ist die Isolierung und Reindarstellung der Urease jedoch nicht erforderlich; es entsprechen die durch Fällung aus dem wässerigen Extrakt gewonnenen Eiweißkörper vollständig ihrem Zweck. Als vorteilhafteste Methode zu deren Gewinnung wird die Fällung durch Eingießen in einen großen Überschuß von Aceton angegeben, wobei sämtliche im wässerigen Extrakt gelösten Eiweißstoffe in groben Flocken ausfallen. Durch Abstehen und Zentrifugieren wird das Aceton entfernt und man erhält so das Ferment in einer für die quantitativen Bestimmungen genügend reinen Form, die außerdem den Vorteil leichterer Wasserlöslichkeit besitzt.

Für die Bestimmungen werden rund 10\% Fermentlösungen verwendet, denen etwas einfach und zweifach saures phosphor- 
saures Kalium zugesetzt wird (auf $1 \mathrm{~g}$ Ferment 0,6 g einfach saures und 0,4 g zweifach saures phosphorsaures Kalium). Diese Salze halten einerseits das Ferment längere Zeit brauchbar und wirken anderseits dem die Fermentwirkung hemmenden Ammoniak entgegen, das bei der Harnstoffzerlegung abgespalten wird. Die hier beschriebene Form der Fermentgewinnung ist von amerikanischen Autoren ${ }^{1}$ ) Donald, D. van Slyke, Got thard Zacharias und Glenn E. Cullen angegeben worden und es sind derartige Fermentlösungen mit Toluol überschichtet gut eine Woche haltbar.

Das Marschallsche Verfahren der quantitativen Analyse wurde von einer Reihe von Autoren modifiziert. Prinzipiell am einfachsten und für rohere Bestimmungen zureichend ist die direkte Titration, wie sie L. J. Geselschab ${ }^{2}$ ) beschreibt, wobei einer gemessenen, stark verdünnten Harnmenge Ferment zugesetzt, verschlossen 24 Stunden aufbewahrt und dann titriert wird. !Die gefundene Alkalescenz vermindert um die des Harnes und des Fermentes entspricht der Menge des bei der Harnstoffzerlegung entstandenen kohlensauren Ammons und daraus läßt sich der Harnstoffgehalt berechnen.

Ich habe mit Harn einige Bestimmungen nach dieser Methode ausgeführt und ziemliche Differenzen verglichen mit dem Pflüger-Schöndorffschen Verfahren gefunden. Die Fehlerquellen liegen wohl hauptsächlich in dem Verlust von freiwerdenden Ammoniak; außerdem ist die Bestimmung der Alkalizität der Fermentlösungen wegen Ausfällung bei Säurezusatz etwas erschwert.

Dagegen ist die Modifikation, wie sie von den oben erwähnten Autoren Donald D. van Slyke usw. in der dort bezeichneten Arbeit mitgeteilt wird, sehr genau und verläßlich. Das Prinzip besteht darin, daß durch einen starken, durchgesaugten Luftstrom, das bei der fermentativen Harnstoffspaltung entstehende Ammoniak in eine Vorlage mit Säure übergetrieben

') Donald van Slyke, Gotthard Zacharias, Glenn E.Kullen, Deutsche med. Wochenschr., Bd. 40, S. 1219, 1914.

2) Geselschab, Zentralblatt für innere Medizin, Nr. 26 (1914) Referat 34. 
und dann durch Titration daraus bestimmt wird. Die Harnstoffzerlegung geht bei Anwendung der beschriebenen Fermentlösung sehr rasch von statten und eine Stunde Einwirkung genügt auf alle Fälle. Allerdings müssen die Harnstoffmengen, die dabei quantitativ zerlegt werden sollen, möglichst kleine sein. Im Durchschnitt nimmt man $5 \mathrm{ccm}$ der zu untersuchenden Flüssigkeit in der maximal ungefähr $0,015 \mathrm{~g}$ Harnstoff gelöst sein sollen, was bei Harn einer durchschnittlich zehnfachen Verdünnung entspricht. In den technischen Einzelheiten habe ich mich größtenteils an die genannten Autoren gehalten und verweise diesbezüglich auf deren Arbeit.

Versuche über die Genauigkeit der Methode habe ich vor allem mit analytisch genau hergestellten Lösungen von reinem, im Exsikkator getrocknetem Harnstoff angestellt und die Resultate mit dem Kjeldahlverfahren verglichen. Wie einige Angaben der folgenden Tabelle zeigen, stimmen die Resultate sehr genau überein. Desgleichen wurden bei Bestimmungen aus Harn Kontrollversuche mit dem Pflüger-Schöndorff und Mörner-Folinschen Verfahren ausgeführt.

\begin{tabular}{c|c|c|c|c|c|c}
\hline $\begin{array}{c}\text { Harnstoff- } \\
\text { lösung } \\
\%\end{array}$ & $\begin{array}{c}\text { Kjeldahl- } \\
\text { verfahren } \\
\%\end{array}$ & $\begin{array}{c}\text { Ferment- } \\
\text { verfahren } \\
\%\end{array}$ & aus Harn & $\begin{array}{c}\text { Ferment- } \\
\text { verfahren } \\
\%\end{array}$ & $\begin{array}{c}\text { Pflüger- } \\
\text { Sch önd orff } \\
\%\end{array}$ & $\begin{array}{c}\text { Mörner- } \\
\text { Folin } \\
\%\end{array}$ \\
\hline 1,201 & 1,2 & 1,2 & $\begin{array}{c}\text { Fieber- } \\
\text { harn } \\
\text { Nephritis- }\end{array}$ & 4,32 & 4,29 & 4,3 \\
$\mathbf{2 , 3 3}$ & 2,328 & 2,326 & $\begin{array}{c}\text { harn } \\
\text { Diabetes- } \\
\text { harn }\end{array}$ & 0,814 & 2,18 & 2,179 \\
& 3 & 3 & 0,821 & 0,79
\end{tabular}

Der große Vorteil dieses modifizierten Marschallschen Verfahrens liegt vor allem in der Schnelligkeit und relativen Einfachheit mit der die Bestimmungen ausgeführt werden können. Eine Stunde wirkt das Ferment, wobei das freie Ammoniak bereits durch den Luftstrom übergetrieben wird, dann saugt man nach Zusatz von Kaliumcarbonat bei Verwendung einer gewöhnlichen Wasserstrahlpumpe den Luftstrom noch etwa eine halbe Stunde durch und titriert.

Weil auf diese Weise auch Ammoniak, das nicht der Harnstoffzerlegung entstammt, mit übergeht, so wird vorgeschlagen, 
außerdem noch ohne Fermentzusatz das freie Ammoniak überzutreiben, zu bestimmen und bei der Umrechnung in Harnstoff abzuziehen. Aber es scheint, wenigstens bei Bestimmungen aus 24stündigem Harn, daß dadurch abermals allerdings kleine Fehlerquellen entstehen, weil auch dann, wenn man den Harn mit Toluol überschichtet, Harnstoff bakteriell zu Ammoniak zerlegt zu werden scheint und dieses \&freie Ammoniak, eigentlich nicht abgezogen werden sollte. Ich fand nämlich bei wachsendem Harnstoffgehalt auch einen beinahe gesetzmäßig wachsenden Gehalt an freiem $\mathrm{NH}_{3}$. Aus einigen Angaben der folgenden Tabelle ist dieses Verhältnis zu erkennen.

\begin{tabular}{|c|c|c|}
\hline & $\begin{array}{c}\text { Tagesmenge Harnstoff } \\
\mathrm{g}\end{array}$ & $\begin{array}{c}\text { Tagesmenge } \mathrm{NH}_{3} \\
\mathbf{g}\end{array}$ \\
\hline Fieberharn . . . . • & 41 & 0,642 \\
\hline Diabetesharn . . . . & · $\quad 36,12$ & 0,577 \\
\hline Norm, Harn . . . . & 32 & 0,512 \\
\hline Norm. Harn . . . . & 28 & 0,481 \\
\hline Norm. Harn . . . . & 26 & 0,402 \\
\hline
\end{tabular}

Es folgt nun eine Zusammenstellung der täglichen Harnstoffausscheidung mit gleichzeitiger Angabe des Gesamtstickstoffs, zunächst bei einem mageren, sonst normalen Individuum von $60 \mathrm{~kg}$ Körpergewicht und etwas geringerer Diurese, bei gleicher Lebensweise mit wenig Körperarbeit.

\begin{tabular}{c|c|c|c}
\hline \hline Norm. Harn & $\begin{array}{c}\text { Harnstoff } \\
\text { in } \\
\%\end{array}$ & $\begin{array}{c}\text { Harnstoff } \\
\text { Tagesmenge } \\
\text { g }\end{array}$ & $\begin{array}{c}\text { Gesamtstickstoff } \\
\text { Tagesmenge } \\
\text { g }\end{array}$ \\
\hline $\begin{array}{c}\text { Menge 1050 } \\
\text { spez. Gew. 1,025 } \\
\text { Menge 1050 } \\
\text { spez. Gew. 1,025 } \\
\text { Menge 900 }\end{array}$ & 2,64 & 27,72 & 16,483 \\
$\begin{array}{c}\text { spez. Gew. 1,024 } \\
\text { Menge 860 } \\
\text { spez. Gew. 1,026 } \\
\text { Menge 980 } \\
\text { spez. Gew. 1,026 } \\
\text { Menge 1000 }\end{array}$ & 3,76 & 28,98 & 15,29 \\
spez. Gew. 1,025 & 2,06 & 24,84 & 15,3 \\
\hline
\end{tabular}


Zum Vergleich dazu ein Diabetes mellitus einer 70jährigen Patientin von $35 \mathrm{~kg}$ Körpergewicht bei Bettruhe.

\begin{tabular}{c|c|c|c|c}
\hline Diabetesharn & $\begin{array}{c}\text { Harnstoff } \\
\text { in } \\
\%\end{array}$ & $\begin{array}{c}\text { Harnstoff } \\
\text { Tagesmenge } \\
\mathbf{g}\end{array}$ & $\begin{array}{c}\text { Gesamtstickstoff } \\
\text { Tagesmenge } \\
\mathbf{g}\end{array}$ & $\begin{array}{c}\text { Harnzacker } \\
\text { Tagesmenge } \\
\mathbf{g}\end{array}$ \\
\hline $\begin{array}{c}\text { Menge 4500 } \\
\text { spez. Gew. 1,031 } \\
\text { Menge 4900 }\end{array}$ & 0,72 & 32,4 & 16,69 & 297 \\
$\begin{array}{c}\text { spez. Gew. 1,030 } \\
\text { Menge 4000 }\end{array}$ & 0,636 & 31,16 & 16,8 & 269,5 \\
$\begin{array}{c}\text { spez. Gew. 1,031 } \\
\text { Menge 5500 }\end{array}$ & 0,72 & 28,8 & 15 & 260 \\
$\begin{array}{c}\text { spez. Gew. 1,030 } \\
\text { Menge 5500 }\end{array}$ & 0,576 & 31,68 & 15,76 & 275 \\
spez. Gew. 1,030 \\
$\begin{array}{c}\text { Menge 3600 } \\
\text { spez. Gew. 1,032 }\end{array}$ & 0,66 & 36,3 & 19,12 & 330 \\
\hline
\end{tabular}

Die spezifische Wirkung des Fermentes gestattet es auch ohne weiteres, aus eiweißhaltigen Flüssigkeiten, wie Blut, Exund Transsudaten, Se- und Exkreten, Harnstoff bestimmungen auszuführen. In 2 Fällen habe ich auch den Reststickstoff bestimmt, wobei zum Enteiweißen die von Philipp ${ }^{1}$ ) in unserer Klinik als genaueste Methode erkannte Uranylazetat-Fällung ausgeführt wurde, und diese Resultate mit den gefundenen Harnstoffwerten verglichen (siehe umstehende Tabelle).

Interessant und für die Anschauungen J. Löwys ${ }^{2}$ ) bedeutungsvoll ist die nahe Übereinstimmung der Harnstoffwerte bei Ascites-Transsudat und Lumbalflüssigkeit, ohne daß jedoch aus diesem einen Resultat ein weiterer Schluß gezogen werden könnte.

Ferner ist aus der Tabelle zu ersehen, daß die Reststickstoffwerte bedeutend größer sind, als die zugehörigen Harnstoffwerte; und diese bewegen sich wiederum an der unteren Grenze der von R. v. Jaksch ${ }^{3}$ ) ermittelten Durchschnittswerte von Harnstoff in Blut.

1) Philipp, Diese Zeitschrift, Bd. 86, S. 494 (1913).

2) J. Löwy, D. Archiv f. klin. Med., Bd. 116, S. 79, 1915.

$\left.{ }^{3}\right)$ R. von Jaksch, Internat. Beitr. z. inn. Med. Festschr. f. Leyden, Berlin 1902, Bd. 97. Zeitschr. f. Heilk., Bd. 24, S. 401, 1903. 
Uber die Urease der Sojabohne und ihre Verwendung.

\begin{tabular}{c|c|c}
\hline & $\begin{array}{c}\text { Harnstoff in } \\
\%\end{array}$ & - \\
\hline $\begin{array}{c}\text { Blutserum (Chlorose) } \\
\text { Blutserum (Atherosklerose) }\end{array}$ & 0,0312 & 0,0494 \\
Blütserum (Typhus abdom.) & 0,0756 & $0,0381 \%$ Reststickstoff \\
$\begin{array}{c}\text { Blutserum } \\
\text { (Nephritis, Hemiplegie) }\end{array}$ & $0,0463 \%$. Reststickstoff \\
$\begin{array}{c}\text { Blutserum } \\
\text { (Schwangerschaftsnephritis) }\end{array}$ & 0,371 & - \\
$\begin{array}{c}\text { Pleuritisches Exsudat } \\
\text { (Pleuritis tubercul.) }\end{array}$ & 0,031 & - \\
$\begin{array}{c}\text { Pleuritisches Exsudat } \\
\text { (Carcinommetastasen in } \\
\text { pleura) }\end{array}$ & 0,043 & - \\
$\begin{array}{c}\text { Ascites-Flüssigkeit } \\
\text { (Cirrhosis hepatis) }\end{array}$ & 0,030 & - \\
$\begin{array}{c}\text { Lumbal-Flüssigkeit } \\
\text { (Meningitis Abc.) } \\
\text { norm. Speichel }\end{array}$ & 0,0301 & - \\
\end{tabular}

Nachdem durch die Fermentmethode nur Harnstoff und dieser quantitativ zerlegt wird, so glaube ich, daß bei den anderen Methoden der Harnstoffbestimmung aus Blut die größeren Werte auf Stickstoffșubstanzen zurückzuführen sind, die durch die üblichen Fällungsmethoden nicht entfernt werden können. Vielleicht handelt es sich zum Teil um Kolloidstoffe des Serums, auf deren große Bedeutung für die Ätiologie und Pathologie der Autotoxicosen H. Přibramª ${ }^{1}$ ) hingewiesen hat.

Weitere Studien in dieser. Richtung behalte ich mir für spätere Zeiten vor.

1) H. Přibram, Münchener med. Wochenschr., Bd. 60, S. 2047, 1913 und Zentralbl. f. inn. Med., Bd. 35, S. 7, 1914. 